\title{
Maturing ooctyes: the role of MAP kinase
}

One of the most simple and fundamental features of the eukaryotic cell cycle is that each synthesis $(\mathrm{S})$ phase is followed by a mitosis (M) phase. Hence, DNA replication is followed by cell division. In meiosis, however, this does not occur. After the $M$ phase of the first meiotic division, cells enter into a second meiotic $M$ phase without an intervening $S$ phase. This lack of $S$ phase ensures that four haploid cells will be derived from the diploid mother cell. The processes that control this have been most extensively studied in maturing Xenopus oocytes. Here, the hormone progesterone drives the immature oocyte into meiosis I, after which the metaphase division of meiosis II occurs consecutively, with no interim S phase.

To ensure that the cell drives straight into meiosis II after meiosis I, high levels of cyclin B must be maintained, and Mos (a mitogen-activated kinase kinase kinase) must be active. Low levels of cyclin B usually trigger S phase, whereas the activity of wild-type Mos can suppress S phase. After meiosis I, levels are cyclin B are kept unusually high, as only $50 \%$ of the protein is targeted for degradation by the anaphase-promoting complex (APC). This, combined with an increase in its synthesis, ensures that cyclin B levels are high enough to trigger meiosis II. This apparent abundance of cyclin $\mathrm{B}$, and the lack of its degradation, is thought to be due to changes in the activity of the APC component Cdc27. However, the precise function of mitogen-activated protein kinase (MAPK; a downstream activator of Mos) in this maturation process was unknown. This was partly because of the lack of a potent MAPK inhibitor for experimental use.

In a recent paper, however, Gross et al. (Curr. Biol. 10, 430-438, 2000) have identified a new inhibitor of MAPK, U1026, which has $100 \%$ efficacy. Using this inhibitor, they have shown that when MAPK is completely inhibited, Xenopus oocytes can still enter meiosis I, as in the wild type. However, instead of maturing and entering meiosis II, the cells then proceed directly into $S$ phase. High levels of cyclin B are not maintained in these oocytes. There is also no phosphorylation of Cdc27 (meaning that the APC is not inacti-
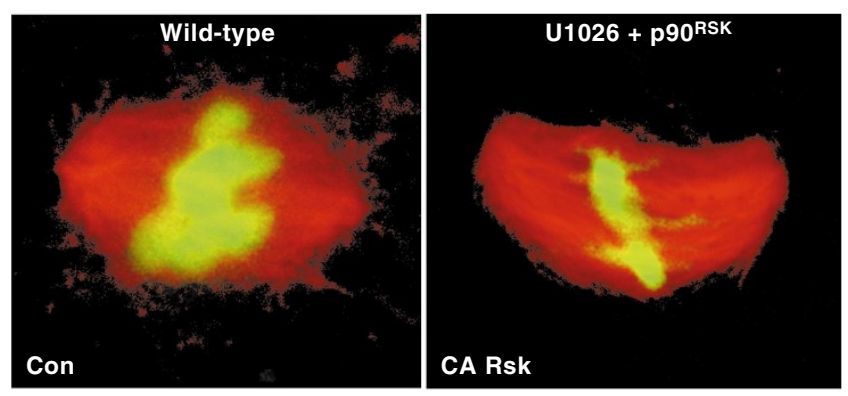

vated) and no reorganization of the meiotic spindle that would allow meiosis II to occur. However, if a downstream activator of MAPK, p90rsk, is added to these oocytes, cyclin B levels rise, spindles form and Cdc27 is phosphorylated (picture shows reorganization of the meiotic spindle).

These experiments have allowed the precise function of MAPK in oocyte maturation to be determined. It seems that, contrary to popular expectation, activation of MAPK is not essential for the entry of oocytes into meiosis I. However, its activation is clearly essential to ensure that oocytes enter the second meiotic division rather than embarking on an $S$ phase.

These activities of MAPK, which are probably mediated by p90rsk, allow the accumulation of large amounts of cyclin B and the regulation of the APC to prevent degradation. MAPK also promotes spindle reorganization to support meiosis II and not $S$ phase. Future work will now focus on determining the intervening steps between MAPK, the APC and control of the meiotic spindle. It seems that the understanding of MAPK's influence on meiosis II is far from being 100\% mature. 